\title{
Twistorial maps between quaternionic manifolds
}

\author{
Stere IanuŞ, Stefano Marchiafava, Liviu Ornea And Radu Pantilie
}

This paper is dedicated to the memory of Kris Galicki

\begin{abstract}
We introduce a natural notion of quaternionic map between almost quaternionic manifolds and we prove the following, for maps of rank at least one:

- A map between quaternionic manifolds endowed with the integrable almost twistorial structures is twistorial if and only if it is quaternionic.

- A map between quaternionic manifolds endowed with the nonintegrable almost twistorial structures is twistorial if and only if it is quaternionic and totallygeodesic.
\end{abstract}

As an application, we describe all the quaternionic maps between open sets of quaternionic projective spaces.

Mathematics Subject Classification (2010): 53C28 (primary); 53C26 (secondary).

\section{Introduction}

An almost quaternionic structure on a manifold is a reduction of its frame bundle to the group $\operatorname{Sp}(1) \cdot \operatorname{GL}(m, \mathbb{H})$. The integrability condition for an almost quaternionic structure (that is, the condition that the corresponding reduction of the frame bundle be given by the cocycle determined by an atlas) is very restrictive [15]; nevertheless there exists only one more general notion of integrability, which amounts to the existence of a compatible torsion free connection (see [21]). In dimension at least eight, such a connection is called quaternionic whilst, in dimension four, a quaternionic connection is a Weyl connection whose Weyl curvature tensor is anti-self-

S.I., L.O. and R.P. acknowledge that this work was partially supported by a CEx Grant no. 2CEx 06-11-22/25.07.2006; also, R.P. acknowledges that this work was partially supported by a C.N.C.S.I.S. grant, code 811 , and by the Visiting Professors Programme of GNSAGA-INDAM of C.N.R. (Italy). S.M. acknowledges that this work was done under the program of GNSAGAINDAM of C.N.R. and PRIN05 "Geometria riemanniana e strutture differenziabili" of MIUR (Italy).

Received September 15, 2008; accepted March 19, 2009. 
dual. A quaternionic manifold is a manifold endowed with an almost quaternionic structure and a (compatible) quaternionic connection.

It is a basic fact that the problem of the existence of a quaternionic connection on a manifold, endowed with an almost quaternionic structure, admits a twistorial interpretation (see Remark 2.10(2) below).

In this paper we introduce a natural notion of quaternionic map (Definition 2.4) with respect to which the class of quaternionic manifolds becomes a category. Furthermore, we show that the quaternionic maps, of rank at least one, are twistorial in a natural way; that is, they are characterised by the existence of a holomorphic lift between the corresponding twistor spaces (Theorem 3.5).

The paper is organised as follows. In Section 1 we review some facts about quaternionic vector spaces (see [3]). In Section 2, after recalling the definition of almost quaternionic structure, we introduce the notion of quaternionic map and we prove its first properties (Proposition 2.6). Also, in Section 2 we recall the two almost twistorial structures associated to a quaternionic manifold, one of which (Example 2.9) is integrable, whilst the other one (Example 2.11) is nonintegrable.

In Section 3 we study twistorial maps between quaternionic manifolds. Besides the above mentioned relation between quaternionic and twistorial maps, with respect to the (integrable) twistorial structures, we prove that a map, of rank at least one, is twistorial, with respect to the nonintegrable almost twistorial structures, if and only if it is quaternionic and totally geodesic (Theorem 3.7). Another result we obtain is that any quaternionic map is real-analytic, at least, outside the frontier of the zero set of its differential (Corollary 3.6).

Examples of quaternionic maps are given in Section 4. There we also apply results of Section 3 to describe all the quaternionic maps between open sets of quaternionic projective spaces (Theorem 4.5).

Finally, in the Appendix we discuss how the quaternionic maps are related to other, more or less similar, notions. We conclude that the quaternionic maps are the natural morphisms of Quaternionic Geometry.

ACKNOWLEDGEMENTS. We are grateful to the referee for very useful comments and suggestions (in particular, for the alternative proof of Lemma 4.6).

\section{Quaternionic vector spaces}

In this section, we review some facts, from [3], on quaternionic vector spaces and quaternionic linear maps. Unless otherwise stated, all the vector spaces and linear maps are assumed real.

Definition 1.1. Let $A$ and $B$ be (real or complex, unital) associative algebras. Two morphisms $\rho, \sigma: A \rightarrow B$ are called A-equivalent if there exists an automorphism $\tau: A \rightarrow A$ such that $\sigma=\rho \circ \tau$. 
Let $\mathbb{H}$ be the division algebra of quaternions. The group of automorphisms of $\mathbb{H}$ is $\operatorname{SO}(3)$, acting trivially on 1 and canonically on $\operatorname{Im} \mathbb{H}\left(=\mathbb{R}^{3}\right)$; note that all the automorphisms of $\mathbb{H}$ are inner.

The following definition is due to [3].

\section{Definition 1.2.}

1. A linear hypercomplex structure on a vector space $V$ is a morphism of associative algebras from $\mathbb{H}$ to $\operatorname{End}(V)$. A vector space endowed with a linear hypercomplex structure is called a hypercomplex vector space.

2. A linear quaternionic structure on a vector space $V$ is an equivalence class of $\mathbb{H}$-equivalent morphisms of associative algebras from $\mathbb{H}$ to End $(V)$. Any representative of the class defining a linear quaternionic structure is called an admissible linear hypercomplex structure (of the given linear quaternionic structure). A vector space endowed with a linear quaternionic structure is called a quaternionic vector space.

Obviously, a hypercomplex vector space is just a left $\mathbb{H}$-module.

Example 1.3. The natural structure of left $\mathbb{H}$-module on $\mathbb{H}^{m}$ gives the (natural) linear hypercomplex structure of $\mathbb{H}^{m},(m \geq 0)$. Moreover, any hypercomplex vector space is $\mathbb{H}$-linearly isomorphic to $\mathbb{H}^{m}$, for some $m \geq 0$.

The linear hypercomplex structure of $\mathbb{H}^{m}$ determines the (natural) linear quaternionic structure of $\mathbb{H}^{m}$.

A hypercomplex linear map $f: V \rightarrow W$ between hypercomplex vector spaces is an $\mathbb{H}$-linear map.

Let $V$ be a quaternionic vector space and let $\rho: \mathbb{H} \rightarrow$ End $(V)$ be an admissible linear hypercomplex structure. As $\mathrm{SO}(3)$ acting on $\mathbb{H}$, preserves 1 and $\mathrm{Im} \mathbb{H}$, the vector spaces $Q_{V}=\rho(\operatorname{Im} \mathbb{H})$ and $\widetilde{Q}_{V}=\rho(\mathbb{H})$ depend only on the linear quaternionic structure induced by $\rho$ on $V$. Furthermore, $\widetilde{Q}_{V} \subseteq \operatorname{End}(V)$ is a division algebra (noncanonically) isomorphic to $\mathbb{H}$ and $Q_{V}$ is a three-dimensional oriented Euclidean vector space for which any oriented orthonormal basis $(I, J, K)$ satisfies the quaternionic identities (that is, $I^{2}=J^{2}=K^{2}=I J K=-\operatorname{Id}_{V}$ ). Similarly, the unit sphere $Z_{V}=\rho\left(S^{2}\right)$ is well-defined.

Definition 1.4 (cf. [3]). Let $V$ and $W$ be quaternionic vector spaces and let $t: V \rightarrow$ $W$ and $T: Z_{V} \rightarrow Z_{W}$ be maps.

We say that $t$ is a quaternionic linear map, with respect to $T$, if $t$ is linear and

$$
t \circ J=T(J) \circ t
$$

for any $J \in Z_{V}$.

Proposition 1.5. Let $V$ and $W$ be quaternionic vector spaces and let $t: V \rightarrow W$ be a nonzero linear map.

(i) If $t$ is quaternionic linear, with respect to $T_{1}, T_{2}: Z_{V} \rightarrow Z_{W}$, then $T_{1}=T_{2}$.

(ii) If $t$ is quaternionic linear, with respect to some map $T: Z_{V} \rightarrow Z_{W}$, then $T$ can be uniquely extended to an orientation preserving linear isometry from $Q_{V}$ to $Q_{W}$. 
Proof. Let $J \in Z_{V}$. As $t \circ J=T_{k}(J) \circ t,(k=1,2)$, and $t \neq 0$ we have that the kernel of $T_{1}(J)-T_{2}(J)$ is nonzero. But $T_{1}(J)-T_{2}(J)$ is in $\widetilde{Q}_{W}$ which is a division algebra. Thus $T_{1}(J)=T_{2}(J)$. This proves assertion (i).

To prove (ii) we, firstly, obtain, as above, that if $(I, J, K)$ satisfy the quaternionic identities then, also, $(T(I), T(J), T(K))$ satisfy the quaternionic identities.

Now, let $(a, b, c) \in S^{2}$. Then $t \circ(a I+b J+c K)=T(a I+b J+c K) \circ t$. On the other hand, we have

$t \circ(a I+b J+c K)=a t \circ I+b t \circ J+c t \circ K=(a T(I)+b T(J)+c T(K)) \circ t$.

Thus $T(a I+b J+c K) \circ t=(a T(I)+b T(J)+c T(K)) \circ t$ which, because $t \neq 0$, implies that $T(a I+b J+c K)=a T(I)+b T(J)+c T(K)$. The result follows.

Next, we prove the following:

\section{Proposition $1.6([3])$.}

(i) For any quaternionic vector space $V$ there exists a quaternionic linear isomorphism from $V$ to $\mathbb{H}^{m}$ (endowed with its natural linear quaternionic structure), for some $m \geq 0$.

(ii) Any quaternionic linear map $t: \mathbb{H}^{m} \rightarrow \mathbb{H}^{n}$ is given by $t(X)=a X A,(X \in$ $\left.\mathbb{H}^{m}\right)$, for some $a \in \mathbb{H}$ and an $m \times n$ matrix $A$, whose entries are quaternions.

Proof. Assertion (i) follows quickly from the fact that any hypercomplex vector space is $\mathbb{H}$-linearly isomorphic to $\mathbb{H}^{m}$, for some $m \geq 0$.

Let $t: \mathbb{H}^{m} \rightarrow \mathbb{H}^{n}$ be a quaternionic linear map, with respect to some map $T: S^{2}\left(=Z_{\mathbb{H}^{m}}\right) \rightarrow S^{2}\left(=Z_{\mathbb{H}^{n}}\right)$.

If $t=0$ then by taking, for example, $a=1$ and $A=0$ assertion (ii) is trivially satisfied. If $t \neq 0$ then, by Proposition 1.5(ii), there exists $a \in \operatorname{Sp}(1)$ such that $T(\mathrm{i})=a \mathrm{i} a^{-1}, T(\mathrm{j})=a \mathrm{j} a^{-1}, T(\mathrm{k})=a \mathrm{k} a^{-1}$ and one checks immediately that $t^{\prime}=a^{-1} t$ is H-linear.

Let $A$ be the matrix of $t^{\prime}: \mathbb{H}^{m} \rightarrow \mathbb{H}^{m}$ with respect to the canonical bases of the free (left) $\mathbb{H}$-modules $\mathbb{H}^{m}$ and $\mathbb{H}^{m}$. Then $t: \mathbb{H}^{m} \rightarrow \mathbb{H}^{n}$ is given by $t(X)=a X A$, $\left(X \in \mathbb{H}^{m}\right)$, and the proof is complete.

From Proposition 1.6 we obtain the following result.

Corollary 1.7 ([3]). The group of quaternionic linear automorphisms of $\mathbb{H}^{m}$ is equal to $\mathrm{Sp}(1) \cdot \mathrm{GL}(m, \mathbb{H})$.

Let $V$ be a quaternionic vector space and let $\rho: \mathbb{H} \rightarrow \operatorname{End}(V)$ be an admissible linear hypercomplex structure. Obviously, $\rho \otimes \rho: \mathbb{H} \otimes \mathbb{H} \rightarrow \operatorname{End}(V \otimes V)$ is also a morphism of associative algebras (the tensor products are taken over $\mathbb{R}$ ). As $S O(3)$ acts on $\mathbb{H}$ by isometries, $\rho \otimes \rho$ maps the Euclidean structure 
on the (real) dual of $\mathbb{H}$ onto an endomorphism $\beta$ of $V \otimes V$ which depends only of the linear quaternionic structure on $V$. Let $b \in \operatorname{Hom}(V \otimes V, V \odot V)$ be the composition of $\beta$, on the left, with the projection $V \otimes V \rightarrow V \odot V$, where $V \odot V$ is the second symmetric power of $V$. Note that, $b$ is also characterised by

$$
b(X, Y)=\frac{1}{2} \sum_{i=0}^{3}\left(E_{i}(X) \otimes E_{i}(Y)+E_{i}(Y) \otimes E_{i}(X)\right),
$$

for any $X, Y \in V$, where $E_{0}=\rho(1), E_{1}=\rho(\mathrm{i}), E_{2}=\rho(\mathrm{j}), E_{3}=\rho(\mathrm{k})$.

Proposition 1.8. Let $V$ be a quaternionic vector space. For any $J \in Z_{V}$ we denote by $V^{1,0 ; J}$ and $V^{0,1 ; J}$ the eigenspaces of $J$ with respect to $\mathrm{i}$ and $-\mathrm{i}$, respectively.

(i) The subspace $b(V \otimes V)$ of $V \odot V$ is equal to the space of Hermitian contravariant symmetric 2-forms on $V$ (that is, elements of $V \odot V$ invariant under $J \otimes J$, for any $J \in Z_{V}$ ).

(ii) For any $J \in Z_{V}$ and $\alpha \in V^{*}$ we have $\iota_{\alpha}\left(b\left(V^{0,1 ; J}, V^{0,1 ; J}\right)\right)=0$.

(iii) Let $J, K \in Z_{V}$ be orthogonal to each other. Then for any $X \in V^{1,0: J}, Y \in$ $V^{0,1: J}$ and $\alpha \in V^{*}$ we have

$$
\iota_{\alpha}(b(X, Y))=\alpha(X) Y+\alpha(Y) X+\alpha(K X) K Y+\alpha(K Y) K X
$$

Proof. Assertion (i) follows, for example, from relation (1.1).

To prove (ii), let $\alpha \in V^{*}$ and let $I \in Z_{V}$ be included in any admissible hypercomplex basis $(I, J, K)$. Then

$$
\begin{aligned}
2 i_{\alpha}(b(X, Y))= & \alpha(X) Y+\alpha(Y) X+\alpha(I X) I Y+\alpha(I Y) I X \\
& +\alpha(J X) J Y+\alpha(J Y) J X+\alpha(K X) K Y+\alpha(K Y) K X .
\end{aligned}
$$

If $X, Y \in V^{0,1 ; I}$ (that is, $I X=-\mathbf{i} X, I Y=-\mathbf{i} Y$ ) we have

$$
\begin{aligned}
\alpha(I X) I Y+\alpha(I Y) I X & =-(\alpha(X) Y+\alpha(Y) X), \\
\alpha(K X) K Y+\alpha(K Y) K X & =\alpha(I J X) I J Y+\alpha(I J Y) I J X \\
& =-(\alpha(J X) J Y+\alpha(J Y) J X) .
\end{aligned}
$$

The proof of (ii) follows.

Assertion (iii) can be proved similarly.

Remark 1.9. A result similar to Proposition 1.8 can be straightforwardly established for $\beta$. 


\section{Quaternionic manifolds and maps}

In this section we review some basic facts on (almost) quaternionic manifolds (see [3]) and we introduce the notion of quaternionic map.

Unless otherwise stated, all the manifolds and maps are assumed smooth.

Definition 2.1. A (fibre) bundle of associative algebras is a vector bundle whose typical fibre is a (finite-dimensional) associative algebra $A$ and whose structural group is the group of automorphisms of $A$.

Let $E$ and $F$ be bundles of associative algebras. A morphism of vector bundles $\rho: E \rightarrow F$ is called a morphism of bundles of associative algebras if $\rho$ restricted to each fibre is a morphism of associative algebras.

Next, we recall the definitions of almost quaternionic manifolds and almost hypercomplex manifolds.

Definition 2.2 ([5]). An almost quaternionic structure on a manifold $M$ is a pair $(E, \rho)$ where $E$ is a bundle of associative algebras, over $M$, with typical fibre $\mathbb{H}$ and $\rho: E \rightarrow \operatorname{End}(T M)$ is a morphism of bundles of associative algebras. An almost quaternionic manifold is a manifold endowed with an almost quaternionic structure.

An almost hypercomplex structure on a manifold $M$ is an almost quaternionic structure $(E, \rho)$ for which $E=M \times \mathbb{H}$. An almost hypercomplex manifold is a manifold endowed with an almost hypercomplex structure.

It is well-known (see [3]) that there are other ways to define the almost quaternionic and hypercomplex manifolds.

Proposition 2.3. An almost quaternionic structure on a manifold $M$ corresponds to a reduction of the frame bundle of $M$ to $\operatorname{Sp}(1) \cdot \mathrm{GL}(m, \mathbb{H})$ (equivalently, to an $\mathrm{Sp}(1) \cdot \mathrm{GL}(m, \mathbb{H})$-structure $)$.

An almost hypercomplex structure on a manifold $M$ corresponds to a reduction of the frame bundle of $M$ to $\mathrm{GL}(m, \mathbb{H})$.

Proof. Let $M$ be a manifold endowed with an almost quaternionic structure $(E, \rho)$. At each $x \in M$, the morphism $\rho_{x}: E_{x} \rightarrow \operatorname{End}\left(T_{x} M\right)$ determines a structure of quaternionic vector space on $T_{x} M$.

Let $U$ be an open set of $M$ over which $E$ is trivial. Then, by passing to an open subset, if necessary, we can construct a local trivialization $h_{U}: U \times\left.\mathbb{H}^{m} \rightarrow T M\right|_{U}$ of $T M$ which induces quaternionic linear isomorphisms on each fibre.

If $h_{U}$ and $h_{V}$ are two such local trivializations, with $U \cap V \neq \varnothing$, then, by Corollary 1.7, we have that $\left(\left(h_{V}\right)^{-1} \circ h_{U}\right)(x, q)=(x, a(x) q)$ for some map $a$ : $U \cap V \rightarrow \operatorname{Sp}(1) \cdot \mathrm{GL}(m, \mathbb{H}),\left(x \in U \cap V, q \in \mathbb{H}^{m}\right)$.

Conversely, if the frame bundle of $M$ admits a reduction to $\operatorname{Sp}(1) \cdot \operatorname{GL}(m, \mathbb{H})$ then the morphism of Lie groups $\mathrm{Sp}(1) \cdot \mathrm{GL}(m, \mathbb{H}) \rightarrow \mathrm{SO}(3), a \cdot A \mapsto \operatorname{Ad} a$ determines an oriented Riemannian vector bundle $Q$ of rank three and an injective morphism of vector bundles $Q \hookrightarrow \operatorname{End}(T M)$ with the property that any positive 
local orthonormal frame of $Q$ satisfies the quaternionic identities. Let $\widetilde{Q}$ be generated by $Q$ and $\operatorname{Id}_{T M}$. Then $\widetilde{Q} \hookrightarrow \operatorname{End}(T M)$ is a subbundle of associative algebras and its typical fibre is $\mathbb{H}$.

The proof for almost hypercomplex manifolds is similar.

Let $M$ be a manifold endowed with an almost quaternionic structure $(E, \rho)$. Then, as each fibre of $E$ is an associative algebra isomorphic to $\mathbb{H}$, there exists an oriented Riemannian vector subbundle of rank three $\operatorname{Im} E \subseteq E$ with the property that any positive local orthonormal frame of it satisfies the quaternionic identities. Let $Q_{M}=\rho(\operatorname{Im} E)$ and $\widetilde{Q}_{M}=\rho(E)$. Then $\widetilde{Q}_{M} \subseteq \operatorname{End}(T M)$ is a subbundle of associative algebras and its typical fibre is $\mathbb{H}$. Also, $Q_{M}$ is an oriented Riemannian vector bundle of rank three with the property that any positive local orthonormal frame of it satisfies the quaternionic identities; denote by $Z_{M}$ the sphere bundle of $Q_{M}$.

Note that, any almost quaternionic manifold $M,(\operatorname{dim} M=4 m)$, is oriented; at each $x \in M$, the orientation of $T_{x} M$ is given by any $J \in\left(Z_{M}\right)_{x}$. Denote by $L$ the line bundle of $M$; that is, the line bundle over $M$ associated to the frame bundle of positive frames through the morphism of Lie groups $\operatorname{GL}(4 m, \mathbb{R})_{0} \rightarrow(0, \infty)$, $a \mapsto(\operatorname{det} a)^{1 /(4 m)}$; see [14]. (Sometimes, $\left(L^{*}\right)^{4 m}$ is called "the bundle of densities" of $M$ whilst $L$ is called "the bundle of densities of weight 1" or, even, "the weight bundle" of $M$.)

Also, as $\operatorname{Sp}(1)$. GL(1, $\mathbb{H})$ is equal to the connected component of the identity of $\mathrm{CO}(4)$, a four-dimensional almost quaternionic manifold is just an oriented conformal manifold.

Definition 2.4. Let $\varphi: M \rightarrow N$ be a map between almost quaternionic manifolds and let $\Phi: Z_{M} \rightarrow Z_{N}$ be such that $\pi_{N} \circ \Phi=\varphi \circ \pi_{M}$, where $\pi_{M}: Z_{M} \rightarrow M$ and $\pi_{N}: Z_{N} \rightarrow N$ are the projections.

Then $\varphi$ is a quaternionic map, with respect to $\Phi$, if $\mathrm{d} \varphi_{\pi_{M}(J)} \circ J=\Phi(J) \circ$ $\mathrm{d} \varphi_{\pi_{M}(J)}$ for any $J \in Z_{M}$.

A quaternionic immersion/submersion/diffeomorphism is a quaternionic map which is an immersion/submersion/diffeomorphism.

An injective quaternionic immersion is called an almost quaternionic submanifold.

\section{Remark 2.5.}

1. For immersions (and, in particular, diffeomorphisms) our definition of quaternionic map particularizes to give notions already in use (see, for example, [1]).

2. Let $M, N$ and $P$ be almost quaternionic manifolds, and let $\varphi: M \rightarrow N$ and $\psi: N \rightarrow P$ be quaternionic maps, with respect to some maps $\Phi: Z_{M} \rightarrow Z_{N}$ and $\Psi: Z_{N} \rightarrow Z_{P}$, respectively. Then, obviously, $\psi \circ \varphi$ is quaternionic, with respect to $\Psi \circ \Phi$.

The following result follows quickly from Proposition 1.5. 
Proposition 2.6. Let $M$ and $N$ be almost quaternionic manifolds and let $\varphi: M \rightarrow$ $N$ be a map of rank at least one.

(i) If $\varphi$ is quaternionic, with respect to $\Phi_{1}, \Phi_{2}: Z_{M} \rightarrow Z_{N}$, then $\Phi_{1}=\Phi_{2}$.

(ii) If $\varphi$ is quaternionic, with respect to $\Phi: Z_{M} \rightarrow Z_{N}$, then $\Phi$ induces an isomorphism of $\mathrm{SO}(3)$-bundles $Q_{M}=\varphi^{*}\left(Q_{N}\right)$.

Let $M$ be an almost quaternionic manifold, $\operatorname{dim} M=4 m$. An almost quaternionic connection on $M$ is a connection $\nabla$ which induces a connection on $Q_{M}$ (that is, if $J$ is a section of $Q_{M}$ and $X$ is a vector field on $M$ then $\nabla_{X} J$ is a section of $Q_{M}$ ); equivalently, $\nabla$ induces a connection on the reduction to $\operatorname{Sp}(1) \cdot \mathrm{GL}(m, \mathbb{H})$ of the frame bundle of $M$, corresponding to the almost quaternionic structure. If $m \geq 2$, a quaternionic connection on $M$ is a torsion-free almost quaternionic connection. If $m=1$, a quaternionic connection on $M$ is a Weyl connection whose Weyl curvature tensor is anti-self-dual (it is well known that this condition depends only of the underlying oriented conformal structure; see [14] and the references therein).

Definition 2.7 ([21]; $\boldsymbol{c f}$. [16]). A quaternionic manifold is an almost quaternionic manifold endowed with a quaternionic connection. stood.

The set of quaternionic connections on a quaternionic manifold is well-under-

Proposition 2.8 ([17]; see [3]). Let $M$ be a quaternionic manifold, $\operatorname{dim} M=4 m$. The set of quaternionic connections on $M$ is an affine space, over the vector space of 1-forms on $M$, isomorphic to the affine space of connections on $L:$ if $\alpha$ is the difference between the connections induced on $L^{\frac{2 m}{m+1}}$ by two quaternionic connections $\nabla^{2}$ and $\nabla^{1}$ on $M$ then

$$
\nabla_{X}^{2} Y=\nabla_{X}^{1} Y+\alpha(X) Y+\alpha(Y) X-\iota_{\alpha}(b(X, Y)),
$$

for any vector fields $X$ and $Y$ on $M$.

Next, we recall the natural "almost twistorial structures" of a quaternionic manifold (see [14] for the general notion of "almost twistorial structure").

Example 2.9 ([21]). Let $M$ be a quaternionic manifold. The quaternionic connection of $M$ induces a connection $\mathscr{H} \subseteq T Z_{M}$ on $Z_{M}$. Let $\mathscr{H}^{1,0}$ be the complex subbundle of $\mathscr{H}^{\mathbb{C}}$ such that $\mathrm{d} \pi_{M}\left(\mathscr{H}_{J}^{1,0}\right)$ is the eigenspace corresponding to $\mathrm{i}$ of $J \in \operatorname{End}\left(T_{\pi_{M}(J)} M\right)$, for any $J \in Z_{M}$, where $\pi_{M}: Z_{M} \rightarrow M$ is the projection.

Let $\mathcal{J}_{M}$ be the almost complex structure on $Z_{M}$ whose eigenbundle corresponding to $\mathrm{i}$ is equal to $\mathscr{H}^{1,0} \oplus\left(\operatorname{ker} \mathrm{d} \pi_{M}\right)^{1,0}$.

We have that $\mathcal{J}_{M}$ is integrable (this can be proved by using [18, Theorem 1.1]). Furthermore, $\mathcal{J}_{M}$ does not depend of the quaternionic connection on $M$ (this can be proved by using Propositions 1.8(ii) and 2.8).

We call $\tau_{M}=\left(Z_{M}, M, \pi_{M}, \mathcal{J}_{M}\right)$ the twistorial structure of $M$. 
From the integrability of $\tau_{M}$ it follows that there exists a unique real-analytic structure on $M$ with respect to which the following conditions are satisfied:

a) The almost quaternionic structure of $M$ is real-analytic;

b) Locally, there exist real-analytic quaternionic connections on $M$.

Note that, the given quaternionic connection on $M$ is not necessarily real-analytic.

Remark 2.10 ( $c f$. [4]). Let $M$ be an almost quaternionic manifold and let $\nabla$ be an almost quaternionic connection on $M$. Then, similarly to Example 2.9, we construct an almost complex structure $\mathcal{J}^{\nabla}$ on $Z_{M}$ and an almost twistorial structure $\tau^{\nabla}=$ $\left(Z_{M}, M, \pi_{M}, \mathcal{J}^{\nabla}\right)$.

1. Let $J$ be a (local) admissible almost complex structure on $M$ and let $s^{J}$ be the section of $Z_{M}$ corresponding to $J$. Then any two of the following assertions imply the third:

(i) $J$ is integrable;

(ii) $s^{J}:(M, J) \rightarrow\left(Z_{M}, \mathcal{J}^{\nabla}\right)$ is holomorphic;

(iii) $T^{\nabla}\left(\Lambda^{2}\left(T^{0,1 ; J} M\right)\right) \subseteq T^{0,1 ; J} M$, where $T^{\nabla}$ is the torsion of $\nabla$.

2. The almost twistorial structure $\tau^{\nabla}$ is integrable if and only if $M$ admits a quaternionic connection and $\tau^{\nabla}=\tau_{M}$. Indeed, if $\mathcal{J}^{\nabla}$ is integrable then, locally, there exist many admissible almost complex structures $J$ which satisfy assertion (ii), above; moreover, $\mathcal{J}^{\nabla}$ integrable implies that condition (iii) above is satisfied (apply [18, Theorem 1.1]) and therefore any such $J$ is integrable. We have thus proved that if $\mathcal{J}^{\nabla}$ is integrable then, locally, there exist many admissible complex structures on $M$; consequently, $M$ admits a quaternionic connection (if $\operatorname{dim} M=4$ this follows from [22] whilst, if $\operatorname{dim} M \geq 8$, this is a consequence of [4, Theorem 2.4]). Also, we have that $T^{1,0 ; \mathcal{J}^{\nabla}} Z_{M}$ is, pointwisely, generated by $(\operatorname{ker} \mathrm{d} \pi)^{1,0}$ and the holomorphic tangent bundles to the images of the local sections of $Z_{M}$ corresponding to admissible local complex structures on $M$. As this, also, holds for any quaternionic connection on $M$, we obtain $\mathcal{J}^{\nabla}=\mathcal{J}_{M}$.

Example 2.11 (cf. [7]). With the same notation as in Example 2.9, let $\mathcal{J}_{M}^{\prime}$ the almost complex structure on $Z_{M}$ whose eigenbundle corresponding to $\mathrm{i}$ is equal to $\mathscr{H}^{1,0} \oplus\left(\operatorname{ker} \mathrm{d} \pi_{M}\right)^{0,1}$.

We have that $\mathcal{J}_{M}^{\prime}$ is nonintegrable (that is, always not integrable). Furthermore, from Propositions 1.8(iii) and 2.8 it follows that $\mathcal{J}_{M}^{\prime}$ determines the quaternionic connection on $M$. of $M$.

We call $\tau_{M}^{\prime}=\left(Z_{M}, M, \pi_{M}, \mathcal{J}_{M}^{\prime}\right)$ the nonintegrable almost twistorial structure

We end this section with a well-known fact which will be used later on; for the reader's convenience we also supply a proof.

Proposition 2.12 (see [2]). Any almost quaternionic submanifold $N$ of a quaternionic manifold $M$ is totally-geodesic with respect to any quaternionic connection $\nabla$ on $M$. Moreover, $\nabla$ induces a quaternionic connection on $N$. 
Proof. We have $J\left(T_{\pi_{M}(J)} N\right) \subseteq T_{\pi_{M}(J)} N$, for any $\left.J \in\left(Q_{M}\right)\right|_{N}$, where $\pi_{M}$ : $Q_{M} \rightarrow M$ is the projection. Thus, the vector bundle $\left(\left.T M\right|_{N}\right) / T N$ admits a unique reduction to $\mathrm{Sp}(1) \cdot \mathrm{GL}(m-n, \mathbb{H})$ such that the projection $\pi: T M \rightarrow\left(\left.T M\right|_{N}\right) / T N$ is quaternionic linear on each fibre, where $\operatorname{dim} N=4 n$ and $\operatorname{dim} M=4 m$.

Let $B$ be the "second fundamental form" of $N \subseteq M$ with respect to $\nabla$; that is, $B(X, Y)=\pi\left(\nabla_{X} Y\right)$, for any vector fields $X, Y$ on $N$. We have to prove that $B=0$.

Let $J$ be a section of $Z_{M}$ over some open set $U$ of $M$ which intersects $N$. Then, for any $x \in U \cap N$ and $X \in T_{x} N$, we have that $\nabla_{X} J \in\left(Q_{M}\right)_{x}$ and, consequently, $\left(\nabla_{X} J\right)\left(T_{x} N\right) \subseteq T_{x} N$. Therefore, $\nabla_{X}(J Y)-J\left(\nabla_{X} Y\right)$ is a vector field on $U \cap N$, for any vector fields $X, Y$ on $U \cap N$.

Hence, for any $\left.J \in\left(Z_{M}\right)\right|_{N}$ and $X, Y \in T N$, we have $B(X, J Y)=J B(X, Y)$; as $B$ is symmetric, this is equivalent to $B(J X, J Y)=-B(X, Y)$. By applying this property to a positive orthonormal frame of $Q_{M}$ the proof follows quickly.

Remark 2.13. Proposition 2.12 motivates the use of the term "quaternionic submanifold", instead of "almost quaternionic submanifold", when dealing with an ambient quaternionic manifold.

\section{Twistorial maps between quaternionic manifolds}

The following definition is a particular case of [14, Definition 4.1].

Definition 3.1. Let $M$ and $N$ be quaternionic manifolds and let $\varphi: M \rightarrow N$ be a map. Suppose that there exists a map $\Phi: Z_{M} \rightarrow Z_{N}$ such that $\pi_{N} \circ \Phi=\varphi \circ \pi_{M}$.

Denote by $\tau_{M}=\left(Z_{M}, M, \pi_{M}, \mathcal{J}_{M}\right)$ and $\tau_{N}=\left(Z_{N}, N, \pi_{N}, \mathcal{J}_{N}\right)$ the twistorial structures of $M$ and $N$, respectively. Then $\varphi:\left(M, \tau_{M}\right) \rightarrow\left(N, \tau_{N}\right)$ is a $\left(\mathcal{J}\right.$-)twistorial map, with respect to $\Phi$, if $\Phi:\left(Z_{M}, \mathcal{J}_{M}\right) \rightarrow\left(Z_{N}, \mathcal{J}_{N}\right)$ is holomorphic.

Similarly, $\varphi:\left(M, \tau_{M}^{\prime}\right) \rightarrow\left(N, \tau_{N}^{\prime}\right)$ is a $\mathcal{J}^{\prime}$-twistorial map, with respect to $\Phi$, if $\Phi:\left(Z_{M}, \mathcal{J}_{M}^{\prime}\right) \rightarrow\left(Z_{N}, \mathcal{J}_{N}^{\prime}\right)$ is holomorphic, where $\tau_{M}^{\prime}=\left(Z_{M}, M, \pi_{M}, \mathcal{J}_{M}^{\prime}\right)$ and $\tau_{N}^{\prime}=\left(Z_{N}, N, \pi_{N}, \mathcal{J}_{N}^{\prime}\right)$ are the nonintegrable almost twistorial structures of $M$ and $N$, respectively.

Let $M$ be an almost quaternionic manifold and let $J \in Z_{M}$. We denote $T^{1,0 ; J} M$ and $T^{0,1: J} M$ the eigenspaces of $J$ corresponding to $\mathrm{i}$ and $-\mathrm{i}$, respectively.

Proposition 3.2. Let $M$ and $N$ be quaternionic manifolds; denote by $\nabla^{M}$ and $\nabla^{N}$ the quaternionic connections of $M$ and $N$, respectively.

Let $\varphi: M \rightarrow N$ be a quaternionic map with respect to some map $\Phi: Z_{M} \rightarrow$ $Z_{N}$; suppose that $\varphi$ is of rank at least one.

Then the following assertions are equivalent:

(i) $\varphi:\left(M, \tau_{M}\right) \rightarrow\left(N, \tau_{N}\right)$ is twistorial, with respect to $\Phi$.

(ii) $(\nabla \mathrm{d} \varphi)\left(T_{\pi_{M}(J)}^{0,1 ; J} M, T_{\pi_{M}(J)}^{0,1 ; J} M\right) \subseteq T_{\varphi\left(\pi_{M}(J)\right)}^{0,1 ; J} N$, for any $J \in Z_{M}\left(=\varphi^{*}\left(Z_{N}\right)\right)$, where $\nabla$ is the connection, on $\operatorname{Hom}\left(T M, \varphi^{*}(T N)\right)$, induced by $\nabla^{M}$ and $\nabla^{N}$. 
(iii) $A(J X) J=J \circ(A(X) J)$, for any $J \in Z_{M}$ and $X \in T_{\pi_{M}(J)} M$, where $A$ is the difference between the connections, on $Q_{M}\left(=\varphi^{*}\left(Q_{N}\right)\right)$, induced by $\nabla^{M}$ and $\nabla^{N}$.

Proof. To prove (i) $\Longleftrightarrow$ (iii), note that it is sufficient to consider $\varphi$ of constant rank (there exists a dense open subset of $M$ such that on each of its connected components $\varphi$ has constant rank). As then, locally, the image of $\varphi$ is a quaternionic submanifold of $N$, Proposition 2.12 implies that we can further assume $\varphi$ submersive.

Let $J_{0} \in Z_{M}$ and let $x_{0}=\pi_{M}\left(J_{0}\right)$. Also, let $S$ be (the image of) a local section of $\varphi$, containing $x_{0}$, such that $T_{x_{0}} S$ is preserved by $J_{0}$. Locally, we may extend $J_{0}$ to a section $J$ of $Z_{M}$, over $S$. Then there exists a local section $\breve{J}$ of $Z_{N}$ such that $J=\varphi^{*}(\breve{J})$ (equivalently, $\Phi \circ J=\breve{J} \circ \varphi$ ); write $\breve{J}_{0}=\breve{J}_{\varphi\left(x_{0}\right)}$.

Now, the differential of $J$ at $x_{0}$ is a complex linear map from $\left(T_{x_{0}} S,\left.J_{0}\right|_{T_{x_{0}}} S\right)$ to $\left(T_{J_{0}} Z_{M},\left(\mathcal{J}_{M}\right)_{J_{0}}\right)$ if and only if $\nabla_{J_{0} X}^{M} J=J_{0} \circ \nabla_{X}^{M} J$, for any $X \in T_{x_{0}} S$. Similarly, the differential of $\breve{J}$ at $\varphi\left(x_{0}\right)$ is a complex linear map from $\left(T_{\varphi\left(x_{0}\right)} N, \check{J}_{0}\right)$ to $\left(T_{\breve{J}_{0}} Z_{N},\left(\mathcal{J}_{N}\right)_{\breve{J}_{0}}\right)$ if and only if $\nabla_{\breve{J}_{0} X}^{N} \check{J}=\check{J}_{0} \circ \nabla_{X}^{N} \check{J}$, for any $X \in T_{\varphi\left(x_{0}\right)} N$.

It follows that $\mathrm{d} \Phi_{J_{0}}:\left(T_{J_{0}} Z_{M},\left(\mathcal{J}_{M}\right)_{J_{0}}\right) \rightarrow\left(T_{\check{J}_{0}} Z_{N},\left(\mathcal{J}_{N}\right)_{\breve{J}_{0}}\right)$ is complex linear if and only if $A\left(J_{0} X\right) J_{0}=J_{0} \circ\left(A(X) J_{0}\right)$, for any $X \in T_{x_{0}} M$.

To prove (ii) $\Longleftrightarrow$ (iii), firstly, note that, by considering $\mathrm{d} \varphi$ as a section of $\operatorname{Hom}\left(T M, \varphi^{*}(T N)\right)$, we have $\mathrm{d} \varphi \circ J=J \circ \mathrm{d} \varphi$, for any $J \in Z_{M}$. By taking the covariant derivative of this equality we obtain

$$
\left(\nabla_{X} \mathrm{~d} \varphi\right) \circ J-J \circ\left(\nabla_{X} \mathrm{~d} \varphi\right)=(A(X) J) \circ \mathrm{d} \varphi,
$$

for any $J \in Z_{M}$ and $X \in T_{\pi_{M}(J)} M$. The proof follows.

Remark 3.3. The method of Proposition 3.2 can be applied in several other contexts. For example, let $\left(M^{m}, c, D\right)$ be a Weyl space, $\operatorname{dim} M=m$, and let $1 \leq r \leq$ $\frac{1}{2} m$. If $r<\frac{1}{2} m$ let $\pi: P \rightarrow M$ be the bundle of skew-adjoint $f$-structures on $\left(M^{m}, c\right.$ ) whose kernels have dimension $m-2 r$ (any $F \in P$ is a skew-adjoint linear map on $\left(T_{\pi(F)} M, c_{\pi(F)}\right)$ such that $F^{3}+F=0$ and $\left.\operatorname{dim}(\operatorname{ker} F)=m-2 r\right)$. If $r=\frac{1}{2} m,(m$ even), let $P$ be the bundle of positive orthogonal complex structures on $\left(M^{m}, c\right)$.

Then $D$ induces a connection $\mathscr{H} \subseteq T P$ on $P$. Define $\mathscr{H}^{0} \subseteq \mathscr{H}$ such that $\mathrm{d} \pi\left(\mathscr{H}_{F}^{0}\right)=T_{\pi(F)}^{0 ; F} M$, where $T_{\pi(F)}^{0 ; F} M$ is the eigenspace of $F$ corresponding to 0 , $(F \in P)$. Also, define $\mathscr{H}^{0,1} \subseteq \mathscr{H}^{\mathbb{C}}$ such that $\mathrm{d} \pi\left(\mathscr{H}_{F}^{0,1}\right)=T_{\pi(F)}^{0,1 ; F} M$, where $T_{\pi(F)}^{0,1 ; F} M$ is the eigenspace of $F$ corresponding to $-\mathrm{i},(F \in P)$.

Let $\mathcal{F}$ be the almost $f$-structure on $P$ whose eigendistributions corresponding to 0 and $-\mathrm{i}$ are $\mathscr{H}^{0}$ and $\mathscr{H}^{0,1} \oplus\left(\operatorname{ker} \mathrm{d} \pi_{M}\right)^{0,1}$, respectively. Then $\tau_{m, r}=$ $(P, M, \pi, \mathcal{F})$ is an almost twistorial structure on $M$; see [18] for the characterisation of the integrability of $\tau_{m, r}$. 
Now, let $\left(M^{2 n}, c_{M}, D^{M}\right)$ and $\left(N^{2 n-1}, c_{N}, D^{N}\right)$ be Weyl spaces; denote by $\tau_{2 n, n}^{M}=\left(P_{M}, M, \pi_{M}, \mathcal{J}\right)$ and $\tau_{2 n-1, n-1}^{N}=\left(P_{N}, N, \pi_{N}, \mathcal{F}\right)$ the corresponding almost twistorial structures.

Let $\varphi:\left(M^{2 n}, c_{M}\right) \rightarrow\left(N^{2 n-1}, c_{N}\right)$ be a horizontally conformal submersion. There exists a unique map $\Phi: P_{M} \rightarrow P_{N}$ such that $\pi_{N} \circ \Phi=\varphi \circ \pi_{M}$ and

$$
\mathrm{d} \varphi\left(T_{\pi_{M}(J)}^{0,1 ; J} M\right)=T_{\pi_{N}(\Phi(J))}^{0 ; \Phi(J)} N \oplus T_{\pi_{N}(\Phi(J))}^{0,1 ; \Phi(J)} N
$$

for any $J \in P_{M}$.

The following assertions are equivalent:

(i) $\varphi:\left(M^{2 n}, \tau_{2 n, n}^{M}\right) \rightarrow\left(N^{2 n-1}, \tau_{2 n-1, n-1}^{N}\right)$ is twistorial, with respect to $\Phi$ (that is, $\Phi:\left(P_{M}, \mathcal{J}\right) \rightarrow\left(P_{N}, \mathcal{F}\right)$ is holomorphic; equivalently, $\mathrm{d} \Phi\left(T^{0,1} P_{M}\right) \subseteq$ $\left.T^{0} P_{N} \oplus T^{0,1} P_{N}\right)$.

(ii) $(D \mathrm{~d} \varphi)\left(T_{\pi_{M}(J)}^{0,1 ; J} M, T_{\pi_{M}(J)}^{0,1 ; J} M\right) \subseteq \mathrm{d} \varphi\left(T_{\pi_{M}(J)}^{0,1 ; J} M\right)$, for any $J \in P_{M}$, where $D$ is the connection, on $\operatorname{Hom}\left(T M, \varphi^{*}(T N)\right)$, induced by $D^{M}$ and $D^{N}$.

In the particular case $n=2$, from the above equivalence it follows quickly the known (see [14] and the references therein) characterisation of twistorial maps from four-dimensional conformal manifolds to three-dimensional Weyl spaces; also, recall that then, if (i) or (ii) holds, $\left(M^{4}, c_{M}\right)$ is anti-self-dual if and only if $\left(N^{3}, c_{N}\right.$, $\left.D^{N}\right)$ is Einstein-Weyl.

If $n \geq 3$ and (i) or (ii) holds then $\left(M^{2 n}, c_{M}\right)$ is flat if and only if $D^{N}$ is the Levi-Civita connection of constant curvature local representatives of $c_{N}$.

Similarly to Proposition 3.2, we obtain the following result.

Proposition 3.4. Let $M$ and $N$ be quaternionic manifolds; denote by $\nabla^{M}$ and $\nabla^{N}$ the quaternionic connections of $M$ and $N$, respectively.

Let $\varphi: M \rightarrow N$ be a quaternionic map with respect to some map $\Phi: Z_{M} \rightarrow$ $Z_{N}$; suppose that $\varphi$ is of rank at least one.

Then the following assertions are equivalent:

(i) $\varphi:\left(M, \tau_{M}^{\prime}\right) \rightarrow\left(N, \tau_{N}^{\prime}\right)$ is $\mathcal{J}^{\prime}$-twistorial, with respect to $\Phi$.

(ii) $(\nabla \mathrm{d} \varphi)\left(T_{\pi_{M}(J)}^{1,0 ; J} M, T_{\pi_{M}(J)}^{0,1 ; J} M\right) \subseteq T_{\varphi\left(\pi_{M}(J)\right)}^{0,1 ; J} N$, for any $J \in Z_{M}\left(=\varphi^{*}\left(Z_{N}\right)\right)$, where $\nabla$ is the connection, on $\operatorname{Hom}\left(T M, \varphi^{*}(T N)\right)$, induced by $\nabla^{M}$ and $\nabla^{N}$.

(iii) $A(J X) J=-J \circ(A(X) J)$, for any $J \in Z_{M}$ and $X \in T_{\pi_{M}(J)} M$, where $A$ is the difference between the connections, on $Q_{M}\left(=\varphi^{*}\left(Q_{N}\right)\right)$, induced by $\nabla^{M}$ and $\nabla^{N}$.

Next, we prove the following result. 
Theorem 3.5. Let $\varphi: M \rightarrow N$ be a map between quaternionic manifolds and let $\Phi: Z_{M} \rightarrow Z_{N}$ be such that $\pi_{N} \circ \Phi=\varphi \circ \pi_{M}$.

If the zero set of the differential of $\varphi$ has empty interior then the following assertions are equivalent:

(i) $\varphi:\left(M, \tau_{M}\right) \rightarrow\left(N, \tau_{N}\right)$ is twistorial, with respect to $\Phi$.

(ii) $\varphi: M \rightarrow N$ is quaternionic, with respect to $\Phi$.

Proof. Obviously, (i) $\Longrightarrow$ (ii). Thus, it is sufficient to prove (ii) $\Longrightarrow$ (i).

Let $F \subseteq M$ be the zero set of the differential of $\varphi$. As $M \backslash F$ is dense in $M$ and $\pi_{M}$ is open, we have $\pi_{M}^{-1}(M \backslash F)$ dense in $Z_{M}$. Thus, we may suppose that, at each point, $\varphi$ has rank at least one.

By Proposition 3.2, it is sufficient to prove that if $\varphi$ is quaternionic then

$$
(\nabla \mathrm{d} \varphi)\left(T_{\pi_{M}(J)}^{0,1 ; J} M, T_{\pi_{M}(J)}^{0,1 ; J} M\right) \subseteq T_{\varphi\left(\pi_{M}(J)\right)}^{0,1 ; J} N
$$

for any $J \in Z_{M}\left(=\varphi^{*}\left(Z_{N}\right)\right)$.

As in the proof of Proposition 3.2, we may suppose $\varphi$ submersive. Denote $\mathscr{V}=\operatorname{ker} \mathrm{d} \varphi$ and let $\mathscr{H}$ be a distribution on $M$, complementary to $\mathscr{V}$, and which is preserved by $Z_{M}$ (for example, let $\mathscr{H}$ be the orthogonal complement of $\mathscr{V}$ with respect to some Hermitian metric on $M)$; as usual, we identify $\mathscr{H}=\varphi^{*}(T N)$.

Let $J$ be an admissible complex structure (locally) defined on $M$. As $\mathscr{V}$ and $\mathscr{H}$ are invariant under $J$, we have decompositions $\mathscr{V}^{\mathbb{C}}=\mathscr{V}^{0,1 ; J} \oplus \mathscr{V}^{1,0 ; J}$ and $\mathscr{H}^{\mathbb{C}}=\mathscr{H}^{0,1 ; J} \oplus \mathscr{H}^{1,0 ; J}$.

Because $\varphi$ is quaternionic, we have $\mathscr{H}^{0,1 ; J}=\varphi^{*}\left(T^{0,1 ; J} N\right)$.

Let $V$ and $X$ be sections of $\mathscr{V}^{0,1 ; J}$ and $T^{0,1 ; J} M$, respectively. As the section of $Z_{M}$ corresponding to $J$ is a holomorphic map from $(M, J)$ to $\left(Z_{M}, \mathcal{J}_{M}\right)$, we have that $\nabla_{V}^{M} X$ is a section of $T^{0,1 ; J} M$. Hence, $(\nabla \mathrm{d} \varphi)(V, X)=-\mathrm{d} \varphi\left(\nabla_{V}^{M} X\right)$ is a section of $\mathscr{H}^{0,1 ; J}$.

From the fact that $\nabla^{M}$ and $\nabla^{N}$ are torsion-free it follows that there exists a section $\alpha$ of $\mathscr{H}^{*}$ such that $(\nabla \mathrm{d} \varphi)(X, Y)=S^{\alpha}(X, Y)$, for any $X, Y \in \mathscr{H}$ (cf. Proposition 2.8). Hence, by Proposition 1.8, $(\nabla \mathrm{d} \varphi)\left(\mathscr{H}^{0,1 ; J}, \mathscr{H}^{0,1 ; J}\right) \subseteq \mathscr{H}^{0,1 ; J}$.

The proof is complete.

From Theorem 3.5 we obtain the following result (which, also, holds for a more general class of twistorial maps).

Corollary 3.6. Any quaternionic map between quaternionic manifolds is real-anal$y$ tic, at least, outside the frontier of the zero set of its differential.

A condition equivalent to assertion (ii) of the following result is used in [11], for maps between quaternionic Kähler manifolds. 
Theorem 3.7. Let $\varphi: M \rightarrow N$ be a map between quaternionic manifolds and let $\Phi: Z_{M} \rightarrow Z_{N}$ be such that $\pi_{N} \circ \Phi=\varphi \circ \pi_{M}$.

If the zero set of the differential of $\varphi$ has empty interior then the following assertions are equivalent:

(i) $\varphi:\left(M, \tau_{M}^{\prime}\right) \rightarrow\left(N, \tau_{N}^{\prime}\right)$ is $\mathcal{J}^{\prime}$-twistorial, with respect to $\Phi$.

(ii) $\varphi$ is quaternionic, with respect to $\Phi$, and the connections on $Q_{M}\left(=\varphi^{*}\left(Q_{N}\right)\right)$, induced by the quaternionic connections of $M$ and $N$, are equal.

(iii) $\varphi$ is a totally geodesic map which is quaternionic, with respect to $\Phi$.

Proof. This can be proved as follows. By Proposition 3.4, we have (ii) $\Longrightarrow$ (i) and (iii) $\Longrightarrow$ (i). Thus, it remains to prove (i) $\Longrightarrow$ (ii),(iii).

If (i) holds then $\varphi$ is quaternionic and, by Theorem 3.5, $\varphi:\left(M, \tau_{M}\right) \rightarrow$ $\left(N, \tau_{N}\right)$ is twistorial. Thus, assertion (iii) of Proposition 3.2 and assertion (iii) of Proposition 3.4 are both satisfied. This shows that (i) $\Longrightarrow$ (ii).

Finally, from Proposition 3.4 it follows quickly that the $(1,1)$-component of $\nabla \mathrm{d} \varphi$ is zero. This implies $\nabla \mathrm{d} \varphi=0$ and the proof is complete.

Next, we explain why, in Theorems 3.5 and 3.7, the assumption on the zero set of the differential of the map cannot be weakened.

Remark 3.8. Let $M$ and $N$ be quaternionic manifolds. Suppose that $Z_{M}=M \times S^{2}$ is the trivial bundle and let $\pi: Z_{M} \rightarrow S^{2}$ be the projection.

Let $y \in N$ and let $T: S^{2} \rightarrow\left(Z_{N}\right)_{y}$ be an orientation preserving isometry. Then the constant map $\varphi: M \rightarrow N, x \mapsto y,(x \in M)$, is quaternionic, with respect to $\Phi=T \circ \pi$.

On the other hand, $\varphi$ is twistorial, with respect to $\Phi$, if and only if $M$ is hypercomplex.

Note that Theorems 3.5 and 3.7 hold for any nonconstant real-analytic map (without any assumption on the zero set of the differential of the map).

\section{Examples and further results}

Firstly, we mention that, as any quaternionic submanifold corresponds to an injective quaternionic immersion, in [25] can be found many examples of quaternionic maps. For example, we have the following:

Example 4.1. Let $\mathrm{Gr}_{2}(m+2, \mathbb{C})$ be the Grassmannian manifold of complex vector subspaces, of dimension 2 , of $\mathbb{C}^{m+2},(m \geq 1)$.

This is a quaternionic manifold of (real) dimension $4 m$. Its twistor space is the flag manifold $\mathrm{F}_{1, m+1}(m+2, \mathbb{C})$ of pairs $(l, p)$ with $l$ and $p$ complex vector subspaces of $\mathbb{C}^{m+2}$ of dimensions 1 and $m+1$, respectively, such that $l \subseteq p$. The projection $\pi: \mathrm{F}_{1, m+1}(m+2, \mathbb{C}) \rightarrow \mathrm{Gr}_{2}(m+2, \mathbb{C})$ is defined by $\pi(l, p)=l \oplus p^{\perp}$, for any $(l, p) \in \mathrm{F}_{1, m+1}(m+2, \mathbb{C})$, where $p^{\perp}$ is the orthogonal complement of $p$ with respect to the canonical Hermitian product on $\mathbb{C}^{m+2}$. 
Any injective complex linear map $A: \mathbb{C}^{m+2} \rightarrow \mathbb{C}^{n+2},(1 \leq m \leq n)$, induces, canonically, a quaternionic map $\varphi^{A}: \mathrm{Gr}_{2}(m+2, \mathbb{C}) \rightarrow \mathrm{Gr}_{2}(n+2, \mathbb{C})$, with respect to the map $\Phi^{A}: \mathrm{F}_{1, m+1}(m+2, \mathbb{C}) \rightarrow \mathrm{F}_{1, n+1}(n+2, \mathbb{C})$ defined by $\Phi^{A}(l, p)=$ $(A(l), A(p) \oplus q)$, for any $(l, p) \in \mathrm{F}_{1, m+1}(m+2, \mathbb{C})$, where $q \subseteq \mathbb{C}^{n+2}$ is a fixed complement of $\operatorname{im} A$ in $\mathbb{C}^{n+2}$. (Note that, if we choose another complement of $\operatorname{im} A$ in $\mathbb{C}^{n+2}$ then $\Phi^{A}$ changes by a composition, to the left, with a holomorphic diffeomorphism of $\mathrm{F}_{1, n+1}(n+2, \mathbb{C})$.)

The next example shows that, besides quaternionic immersions, there are many other quaternionic maps.

Example 4.2. Let $\mathbb{H} P^{m}$ be the left quaternionic projective space of (real) dimension $4 m,(m \geq 1)$. This is a quaternionic manifold (see [15]). Its twistor space is $\mathbb{C} P^{2 m+1}$, where the projection $\pi: \mathbb{C} P^{2 m+1} \rightarrow \mathbb{H} P^{m}$ is induced by the identification $\mathbb{H}^{m+1}=\mathbb{C}^{2 m+2}$, through the morphism of Lie groups $\mathbb{C}^{*} \rightarrow \mathbb{H}^{*}$.

Let $A: \mathbb{H}^{m+1} \rightarrow \mathbb{H}^{n+1}$ be a left $\mathbb{H}$-linear map, $(m, n \geq 1)$. Then $A$ induces two maps $\varphi^{A}: \mathbb{H} P^{m} \backslash P_{\mathbb{H}}(\operatorname{ker} A) \rightarrow \mathbb{H} P^{n}$ and $\Phi^{A}: \mathbb{C} P^{2 m+1} \backslash P_{\mathbb{C}}(\operatorname{ker} A) \rightarrow$ $\mathbb{C} P^{2 n+1}$, where $P_{\mathbb{H}}(\operatorname{ker} A)$ and $P_{\mathbb{C}}(\operatorname{ker} A)$ are the quaternionic and complex projective spaces, respectively, determined by $\operatorname{ker} A$.

Then $\varphi^{A}$ is a quaternionic map, with respect to $\Phi^{A}$ (just note, for example, that $\Phi^{A}$ is holomorphic).

Example 4.3. Let $\pi: \mathbb{H}^{m+1} \backslash\{0\} \rightarrow \mathbb{H} P^{m}$ be the Hopf fibration. Then $\pi$ is a quaternionic map, with respect to the canonical projection

$$
\Pi: 2(m+1) \mathcal{O}(1) \backslash 0 \rightarrow P(2(m+1) \mathcal{O}(1))=\mathbb{C} P^{2 m+1},
$$

where $\mathcal{O}(1)$ is the dual of the tautological line bundle over $\mathbb{C} P^{1}$.

The following example based on a construction of [24] (see [19]) is, essentially, a generalization of Example 4.3.

Example 4.4. Let $M$ be a quaternionic manifold of dimension $4 m,(m \geq 1)$, and let $(P, M, \operatorname{Sp}(1) \cdot \mathrm{GL}(m, \mathbb{H}))$ be its bundle of quaternionic frames (that is, quaternionic linear isomorphisms from $\mathbb{H}^{m}$ to $\left.T_{x} M,(x \in M)\right)$.

Define $\rho: \operatorname{Sp}(1) \cdot \operatorname{GL}(m, \mathbb{H}) \rightarrow \mathbb{H}^{*} /\{ \pm 1\}$ by $\rho(a \cdot A)= \pm a$, for any $a \cdot A \in$ $\operatorname{Sp}(1) \cdot \operatorname{GL}(m, \mathbb{H})$, where $\mathbb{H}^{*}=\mathbb{H} \backslash\{0\}$.

Denote $H=\rho(P)$. Then $\left(H, M, \mathbb{H}^{*} /\{ \pm 1\}\right)$ is a principal bundle. Furthermore, the quaternionic connection of $M$ induces a principal connection $\mathscr{H} \subseteq T H$. Let $\mathscr{V}=\operatorname{ker} \pi$, where $\pi: H \rightarrow M$ is the projection.

Let $q \in S^{2} \subseteq \operatorname{Im} \mathbb{H}$. The multiplication to the right by $-q$ defines a negative (linear) complex structure on $\mathbb{H}$ which, obviously, is invariant under the left action of $\mathbb{H}^{*}$ on $\mathbb{H}$. Thus, $q$ induces on $\mathscr{V}$ a structure of complex vector bundle $J^{q, \mathscr{V}}$. 
As $\widetilde{Q}_{M}$ is a bundle associated to $H$, we have $\pi^{*}\left(\widetilde{Q}_{M}\right)=H \times \mathbb{H}$. Together with the fact that $\mathscr{H}=\pi^{*}(T M)$ this induces a left action of $\mathbb{H}^{*}$ on $\mathscr{H}$. In particular, $q$ induces on $\mathscr{H}$ a structure of complex vector bundle $J^{q, \mathscr{H}}$.

Obviously, $J^{q}=J^{q, \mathscr{V}} \oplus J^{q, \mathscr{H}}$ is an almost complex structure on $H$.

The morphism of Lie groups $\mathbb{C}^{*} \rightarrow \mathbb{H}^{*}$ given by $a+b \mathrm{i} \mapsto a+b q,(a, b \in$ $\mathbb{R})$, induces a right action of $\mathbb{C}^{*} /\{ \pm 1\}$ on $H$. Furthermore, the quotient of $H$ through this action is $Z_{M}$ and the projection $\psi^{q}:\left(H, J^{q}\right) \rightarrow\left(Z_{M}, \mathcal{J}^{M}\right)$ is holomorphic.

From the fact that $\mathcal{J}^{M}$ is integrable it follows that $J^{q}$ is integrable. Thus, $\left(J^{\mathrm{i}}, J^{\mathrm{j}}, J^{\mathrm{k}}\right)$ defines a hypercomplex structure on $H$. The complex structure $\mathcal{J}_{H}$ of its twistor space $Z_{H}\left(=H \times S^{2}\right)$ is characterised by the following: $\left.\mathcal{J}_{H}\right|_{\{e\} \times S^{2}}$ is the canonical complex structure of $S^{2}$ whilst $\left.\mathcal{J}_{H}\right|_{H \times\{q\}}=J^{q}$, for any $e \in H$ and $q \in S^{2}$.

Note that, $Z_{M}=H \times_{\chi} S^{2}$, where $\chi:\left(\mathbb{H}^{*} /\{ \pm 1\}\right) \times S^{2} \rightarrow S^{2}$ is defined by $\chi( \pm p, q)=p q p^{-1}$, for any $\pm p \in \mathbb{H}^{*} /\{ \pm 1\}$ and $q \in S^{2}$. Let $\Pi: Z_{H} \rightarrow Z_{M}$ be the projection. Alternatively, $\Pi$ can be defined by $\Pi(e, q)=\psi^{q}(e)$, for any $(e, q) \in Z_{H}$.

Then $\Pi:\left(Z_{H}, \mathcal{J}_{H}\right) \rightarrow\left(Z_{M}, \mathcal{J}_{M}\right)$ is holomorphic and $\pi: H \rightarrow M$ is a quaternionic submersion, with respect to $\Pi$.

Next, we prove that Example 4.2 gives all the quaternionic maps between open sets of quaternionic projective spaces.

Theorem 4.5. Let $U$ be a connected open set of $\mathbb{H} P^{m}$ and let $\varphi: U \rightarrow \mathbb{H} P^{n}$ be a quaternionic map, $(m, n \geq 1)$.

Then there exists an $\mathbb{H}$-linear map $A: \mathbb{H}^{m+1} \rightarrow \mathbb{H}^{n+1}$ such that $\varphi=\left.\varphi^{A}\right|_{U}$ and, in particular, $U \cap P_{\mathbb{H}}(\operatorname{ker} A)=\emptyset$.

To prove Theorem 4.5, we need two lemmas, the first of which is, most likely, known but we do not have a reference for it.

Lemma 4.6. Let $M$ be a quaternionic manifold and let $\tau_{M}=\left(Z_{M}, M, \pi_{M}, \mathcal{J}_{M}\right)$ be its twistorial structure.

Then a function $f: M \rightarrow \mathbb{C}$ is constant if and only if $f \circ \pi_{M}:\left(Z_{M}, \mathcal{J}_{M}\right) \rightarrow \mathbb{C}$ is holomorphic.

Proof. If $f \circ \pi_{M}:\left(Z_{M}, \mathcal{J}_{M}\right) \rightarrow \mathbb{C}$ is holomorphic then $f$ is holomorphic with respect to any (local) admissible complex structure $J$ on $M$; equivalently, the differential of $f$ is zero on $T^{0,1 ; J} M$.

It follows that $\mathrm{d} f=0$ and the lemma is proved. 
Lemma 4.6 can be also proved by considering the real structure $\sigma: J \mapsto-J$, as $F=f \circ \pi_{M}$ is holomorphic and $F=F \circ \sigma$ is anti-holomorphic. Then $\mathrm{d} F=0$ and, as $\pi_{M}$ is a submersion, $\mathrm{d} f=0$.

Lemma 4.7 (cf. [3]). Let $A$ be a complex linear map from $\mathbb{H}^{m+1}=\mathbb{C}^{2 m+2}$ to $\mathbb{H}^{n+1}=\mathbb{C}^{2 n+2}$, of complex rank at least $4,(m, n \geq 1)$. Suppose that there exists an open set $U \subseteq \mathbb{H}^{m+1}$ such that A maps the intersection of any quaternionic line (through the origin) with $U$ into a quaternionic line.

Then $A$ is quaternionic linear.

Proof. The (germ unique) complexification of $\mathbb{H} P^{m}$ is $\mathrm{Gr}_{2}(2 m+2, \mathbb{C}$ ) (to show this, use the fact that $\mathrm{GL}(m+1, \mathbb{H})$ acts transitively on $\left.\mathbb{H} P^{m}\right)$.

Clearly, $A$ determines a holomophic map $\psi^{A}: \mathfrak{A} \rightarrow\left(\mathbb{H} P^{n}\right)^{\mathbb{C}}$, where $\mathfrak{A}$ is the (open) subset of $\mathrm{Gr}_{2}(2 m+2, \mathbb{C})$ formed of those two-dimensional complex vector subspaces of $\mathbb{C}^{2 m+2}$ whose intersection with $\operatorname{ker} A$ is $\{0\}$. Note that, $\psi^{A}$ has a pole along $\left(\mathbb{H} P^{m}\right)^{\mathbb{C}} \backslash \mathfrak{A}$.

From the hypothesis, it follows that the restriction of $\psi^{A}$ to some open set intertwines the conjugations $C_{m}$ and $C_{n}$ of $\left(\mathbb{H} P^{m}\right)^{\mathbb{C}}$ and $\left(\mathbb{H} P^{n}\right)^{\mathbb{C}}$, respectively. By analyticity, we obtain that $\psi^{A}$ and $C_{n} \circ \psi^{A} \circ C_{m}$ determine a holomorphic map on $\mathfrak{A} \cup C_{m}(\mathfrak{A})$. Consequently, $C_{m}(\mathfrak{A})=\mathfrak{A}$ (otherwise, $\psi^{A}$ could be holomorphically extended over points of $\left.\left(\mathbb{H} P^{m}\right)^{\mathbb{C}} \backslash \mathfrak{A}\right)$ and $C_{n} \circ \psi^{A}=\psi^{A} \circ C_{m}$. Hence, $A$ maps any quaternionic line whose intersection with $\operatorname{ker} A$ is $\{0\}$ onto a quaternionic line.

Note that, there are no quaternionic lines which intersect $\operatorname{ker} A$ along complex vector spaces of dimension 1 (otherwise, the map $\Phi^{A}$ from $\mathbb{C} P^{2 m+1} \backslash P_{\mathbb{C}}(\operatorname{ker} A)$ to $\mathbb{C} P^{2 n+1}$, determined by $A$, would induce a continuous extension of $\psi^{A}$ over points of $\left.\left(\mathbb{H} P^{m}\right)^{\mathbb{C}} \backslash \mathfrak{A}\right)$.

Thus, $A$ maps any quaternionic line into a quaternionic line and, by [3, Theorem 1.1], the proof of the lemma is complete.

Remark 4.8. With the same notation as in Lemma 4.7 and its proof, let $\varphi^{A}: \mathbb{H} P^{m} \backslash$ $P_{\mathbb{H}}(\operatorname{ker} A) \rightarrow \mathbb{H} P^{n}$ be the quaternionic map determined by $A$. Then $\psi^{A}$ is the complexification of $\varphi^{A}$.

Proof of Theorem 4.5. Let $\varphi$ be a quaternionic map from a connected open set of $\mathbb{H} P^{m}$ to $\mathbb{H} P^{n}$.

We, firstly, assume the differential of $\varphi$ nowhere zero.

Then, by Corollary 3.6, $\varphi$ is real-analytic. Therefore, it is sufficient to find an $\mathbb{H}$-linear map $A$ such that $\varphi=\varphi^{A}$ on some open set. Moreover, similarly to the proof of Proposition 3.2, we may assume $\varphi$ submersive, surjective and with connected fibres.

By Theorem 3.5, $\varphi$ is twistorial, with respect to some holomorphic map $\Phi$ between open sets $U$ and $V$ of $\mathbb{C} P^{2 m+1}$ and $\mathbb{C} P^{2 n+1}$, respectively. Also, $U$ and $V$ contain families of projective lines (the twistor lines) which are mapped one onto 
another by $\Phi$. Moreover, as the complexification of $\mathbb{H} P^{m}$ contains many complexquaternionic submanifolds, the preimage through $\Phi$ of a hyperplane of $\mathbb{C} P^{2 n+1}$ (not disjoint from $V$ ) is an open subset of a hyperplane of $\mathbb{C} P^{2 m+1}$.

An argument similar to the one used in [9, page 65] shows that the nonhomogeneous components of $\Phi$ divided by suitable linear functions are constant along the twistor lines. Hence, by Lemma 4.6, these meromorphic functions are constant.

We have thus proved that $\Phi$ is induced by some complex linear map from $\mathbb{C}^{2 m+2}$ to $\mathbb{C}^{2 n+2}$. Then the proof (under the assumption that the differential of $\varphi$ is nowhere zero) follows from Lemma 4.7.

Finally, note that, if an $\mathbb{H}$-linear map $A: \mathbb{H}^{m+1} \rightarrow \mathbb{H}^{n+1}$ induces a nonconstant (quaternionic) map $\varphi^{A}: \mathbb{H} P^{m} \backslash P_{\mathbb{H}}(\operatorname{ker} A) \rightarrow \mathbb{H} P^{n}$ then its real rank is, at least, eight. Hence, at each point, the rank of the differential of $\varphi^{A}$ is at least four. It follows quickly that the differential of any nonconstant quaternionic map, between connected open sets of quaternionic projective spaces, is nowhere zero. The proof is complete.

Remark 4.9. Theorem 4.5 can be, also, proved by applying an inductive argument, based on a result of [15], to show that in terms of non-homogeneous quaternionic projective coordinates $\left(x_{j}\right)_{j=1, \ldots, m}$, and $\left(y_{\alpha}\right)_{\alpha=1, \ldots, n}$, on $\mathbb{H} P^{m}$ and $\mathbb{H} P^{n}$, respectively, any quaternionic map $\varphi: \mathbb{H} P^{m} \rightarrow \mathbb{H} P^{n}$ is given by

$$
y_{\alpha}=\left(x_{j} a_{0}^{j}+a_{0}^{0}\right)^{-1}\left(x_{j} a_{\alpha}^{j}+a_{\alpha}^{0}\right), \quad(\alpha=1, \ldots, n),
$$

where the coefficients $a_{\alpha}^{j}$ are constant quaternions (and the Einstein summation convention is used).

We end this section with the following immediate consequence of Theorem 4.5.

Corollary 4.10. Any (globally defined) quaternionic map from $\mathbb{H} P^{m}$ to $\mathbb{H} P^{n}$ is induced by an injective $\mathbb{H}$-linear map $\mathbb{H}^{m+1} \rightarrow \mathbb{H}^{n+1}$; in particular, $m \leq n$.

\section{Appendix}

\section{A. Comparison with other notions of quaternionicity}

Firstly, we mention the fairly standard notion of "hypercomplex (triholomorphic) map" between almost hypercomplex manifolds. Obviously, any such map is quaternionic with respect to the induced almost quaternionic structures on its domain and codomain.

Secondly, there have been studied maps, between quaternionic Kähler manifolds $M$ and $N$, which pull-back Kähler forms of elements of $Z_{N}$ to (the space of) Kähler forms of elements of $Z_{M}$ (see [13]). As the Kähler forms are nondegenerate, 
this condition applies only to immersions and to constant maps, and, therefore, it is too restrictive (also, the presence of a Riemannian metric is required).

Thirdly, there exists the notion of "regular quaternionic function", of one quaternionic variable, introduced in [8] (see [23] for a modern presentation and further results) and later generalised to maps between hyper-Kähler manifolds (see $[6,10]$ and the references therein):

Definition A.1. Let $V$ and $W$ be quaternionic vector spaces and let $T: Z_{V} \rightarrow Z_{W}$ be an orientation preserving isometry.

We say that a map $t: V \rightarrow W$ is linear Fueter-quaternionic, with respect to $T$, if $t$ is real linear and for some (and, consequently, any) positive orthonormal basis $(I, J, K)$ of $Q_{V}$ we have $t=T(I) \circ t \circ I+T(J) \circ t \circ J+T(K) \circ t \circ K$.

With the same notation as in Definition A.1, let $\mathcal{C}_{T}$ be the endomorphism defined by $\mathcal{C}_{T}(t)=T(I) \circ t \circ I+T(J) \circ t \circ J+T(K) \circ t \circ K,\left(t \in \operatorname{Hom}_{\mathbb{R}}(V, W)\right)$, where $(I, J, K)$ is a positive orthonormal basis of $Q_{V}$. A straightforward calculation shows that $\mathcal{C}_{T}$ does not depend of the positive orthonormal basis $(I, J, K)$ and, in particular, the notion of "Fueter-quaternionic map", between almost quaternionic manifolds, is well-defined.

Furthermore, $\mathcal{C}_{T}$ satisfies the equation $\left(\mathcal{C}_{T}\right)^{2}+2 \mathcal{C}_{T}-3=0$. Let $\mathscr{F}_{T}$ and $\mathscr{Q}_{T}$ be the eigenspaces of $\mathcal{C}_{T}$ corresponding to 1 and -3 , respectively. Then $\operatorname{Hom}_{\mathbb{R}}(V, W)=$ $\mathscr{Q}_{T} \oplus \mathscr{F}_{T}$ and $\mathscr{F}_{T}$ is the space of linear Fueter-quaternionic maps, with respect to $T$, whilst $\mathscr{Q}_{T}$ is the space of linear quaternionic maps, with respect to $T$ [10]. Apparently, this would suggest that Fueter-quaternionic maps are "anti-quaternionic". In fact, by reformulating results mentioned in [6] and [10], the following proposition can be easily obtained.

Proposition A.2. Let $V$ and $W$ be quaternionic vector spaces and let $T$ be an orientation preserving isometry from $Z_{V}$ to $Z_{W}$.

Then, for any line through the origin $d \subseteq Q_{V}$, we have $\mathscr{Q}_{T \circ S_{d}} \subseteq \mathscr{F}_{T}$, where $S_{d}$ is the symmetry in $d$. Moreover, $\mathscr{F}_{T}$ is generated by $\bigcup_{d} \mathscr{Q}_{T \circ S_{d}}$.

Finally, let $U, V$ and $W$ be quaternionic vector spaces and let $T^{\prime}: Z_{U} \rightarrow Z_{V}$ and $T^{\prime \prime}: Z_{V} \rightarrow Z_{W}$ be orientation preserving isometries. Also, let $d \subseteq Q_{V}$ be a line through the origin. If $t^{\prime}: U \rightarrow V$ and $t^{\prime \prime}: V \rightarrow W$ are linear quaternionic maps, with respect to $S_{d} \circ T^{\prime}$ and $T^{\prime \prime} \circ S_{d}$, respectively, then, by Proposition A.2, $t^{\prime}$ and $t^{\prime \prime}$ are Fueter-quaternionic, with respect to $T^{\prime}$ and $T^{\prime \prime}$, respectively. However, $t^{\prime \prime} \circ t^{\prime}$ is Fueter-quaternionic, with respect to $T^{\prime \prime} \circ T^{\prime}$, if and only if $t^{\prime \prime} \circ t^{\prime}=0$. Therefore the Fueter-quaternionic maps are not closed under composition.

Moreover, not all linear quaternionic maps are Feuter-quaternionic; see [12], where, and also in [20], an algebraic approach to Hypercomplex Geometry is developed. 


\section{References}

[1] D. V. AlEKSEEVSKY and S. MARChIAFAVA, Quaternionic-like structures on a manifold: Note 2. Automorphism groups and their interrelations, Atti Accad. Naz. Lincei Cl. Sci. Fis. Mat. Natur. Rend. Lincei (9) Mat. Appl. 4 (1993), 53-61.

[2] D. V. AleKSEEVSKY and S. MARChiAfaVA, A report on quaternionic-like structures on a manifold, In: "Proceedings of the International Workshop on Differential Geometry and its Applications" (Bucharest, 1993), Politehn. Univ. Bucharest Sci. Bull. Ser. A Appl. Math. Phys. 55 (1993), 9-34.

[3] D. V. AleKseevsky and S. Marchiafava, Quaternionic structures on a manifold and subordinated structures, Ann. Mat. Pura Appl. 171 (1996), 205-273.

[4] D. V. Alekseevsky, S. Marchiafava and M. Pontecorvo, Compatible complex structures on almost quaternionic manifolds, Trans. Amer. Math. Soc. 351 (1999), 9971014.

[5] E. Bonan, Sur les G-structures de type quaternionien, Cahiers Topologie Géom. Différentielle 9 (1967), 389-461.

[6] J. CHEN and J. LI, Quaternionic maps between hyperkähler manifolds, J. Differential Geom. 55 (2000), 355-384.

[7] J. EELlS and S. SALAMON, Twistorial construction of harmonic maps of surfaces into four-manifolds, Ann. Scuola Norm. Sup. Pisa Cl. Sci. 12 (1985), 589-640.

[8] R. FUETER, Die Funktionentheorie der Differentialgleichungen $\Delta u=0$ und $\Delta \Delta u=0$ mit vier reellen Variablen, Comment. Math. Helv. 7 (1935), 307-330.

[9] P. GRIFFITHS and J. HARRIs, "Principles of Algebraic Geometry", Wiley Classics Library, John Wiley \& Sons, Inc., New York, 1978.

[10] A. HAYDYS, Nonlinear Dirac operator and quaternionic analysis, Comm. Math. Phys. 281 (2008), 251-261.

[11] S. IANUŞ, R. MAZZOCCO and G. E. VÎLCU, Harmonic maps between quaternionic Kähler manifolds, J. Nonlinear Math. Phys. 15 (2008), 1-8.

[12] D. JoYce, Hypercomplex algebraic geometry, Quart. J. Math. Oxford Ser. (2) 49 (1998), $129-162$.

[13] J. LI and X. ZHANG, Quaternionic maps between quaternionic Kähler manifolds, Math. Z. 250 (2005), 523-537.

[14] E. Loubeau and R. PAntilie, Harmonic morphisms between Weyl spaces and twistorial maps II, Ann. Inst. Fourier (Grenoble), to appear.

[15] S. MARChiAfaVA, Sulle varietà a struttura quaternionale generalizzata, Rend. Mat. 3 (1970), 529-545.

[16] V. OproiU, Almost quaternal structures, An. Ştiinţ. Univ. Al. I. Cuza Iaşi Mat. 23 (1977), 287-298.

[17] V. OpRoIU, Integrability of almost quaternal structures, An. Ştiinţ. Univ. Al. I. Cuza Iaşi Mat. 30 (1984), 75-84.

[18] R. PAntilie, On a class of twistorial maps, Differential Geom. Appl. 26 (2008), 366-376.

[19] H. Pedersen, Y. S. Poon and A. F. SWAnn, Hypercomplex structures associated to quaternionic manifolds, Differential Geom. Appl. 9 (1998), 273-292.

[20] D. QuiLlen, Quaternionic algebra and sheaves on the Riemann sphere, Quart. J. Math. Oxford Ser. (2) 49 (1998), 163-198.

[21] S. Salamon, Differential geometry of quaternionic manifolds, Ann. Sci. École Norm. Sup. 19 (1986), 31-55.

[22] S. Salamon, Special structures on four-manifolds, Riv. Mat. Univ. Parma 17* (1991), $109-123$.

[23] A. Sudbery, Quaternionic analysis, Math. Proc. Cambridge Philos. Soc. 85 (1979), 199-224. 
[24] A. Swann, HyperKähler and quaternionic Kähler geometry, Math. Ann. 289 (1991), 421-450.

[25] H. TASAKI, Quaternionic submanifolds in quaternionic symmetric spaces, Tohoku Math. J. 38 (1986), 513-538.

Universitatea din Bucureşti

Facultatea de Matematică

Str. Academiei n. 14

70109, Bucureşti, România

istere@yahoo.com

Dipartimento di Matematica

Istituto "Guido Castelnuovo"

Sapienza Università di Roma

Piazzale Aldo Moro, 2

I 00185 Roma, Italia

marchiaf@mat.uniroma1.it

Universitatea din Bucureşti

Facultatea de Matematică

Str. Academiei n. 14

70109, Bucureşti, România

and

Institutul de Matematică "Simion Stoilow"

al Academiei Române

C.P. 1-764, 014700, Bucureşti, România

liviu.ornea@imar.ro

Institutul de Matematică "Simion Stoilow"

al Academiei Române

C.P. 1-764, 014700, Bucureşti, România

radu.pantilie@imar.ro 